\title{
Atopic Dermatitis and Respiratory Allergy: What is the Link
}

\author{
Danielle C. M. Belgrave ${ }^{1,2} \cdot$ Angela Simpson $^{1} \cdot$ Iain E. Buchan $^{2} \cdot$ Adnan Custovic $^{1}$
}

Published online: 28 September 2015

(C) Springer Science+Business Media New York 2015

\begin{abstract}
Understanding the aetiology and progression of atopic dermatitis and respiratory allergy may elucidate early preventative and management strategies aimed towards reducing the global burden of asthma and allergic disease. In this article, we review the current opinion concerning the link between atopic dermatitis and the subsequent progression of respiratory allergies during childhood and into early adolescence. Advances in machine learning and statistical methodology have facilitated the discovery of more refined definitions of phenotypes for identifying biomarkers. Understanding the role of atopic dermatitis in the development of respiratory allergy may ultimately allow us to determine more effective treatment strategies, thus reducing the patient and economic burden associated with these conditions.
\end{abstract}

Keywords Atopic dermatitis · Respiratory allergy $\cdot$ Asthma Stratified and personalised medicine strategies $\cdot$ Statistical machine learning $\cdot$ Biomarker identification · Dermatology · Review

This article is part of the Topical Collection on Atopic Dermatitis

Danielle C. M. Belgrave

danielle.belgrave@manchester.ac.uk

1 Centre for Respiratory Medicine and Allergy, Institute of Inflammation and Repair, University of Manchester and University Hospital of South Manchester, Manchester, UK

2 Centre for Health Informatics, Institute of Population Health, University of Manchester, Manchester, UK

\section{Introduction}

Understanding the aetiology and progression of atopic dermatitis and respiratory allergy may elucidate early preventative and management strategies aimed towards reducing the global burden of asthma and allergic disease [1,2]. There is increasing interest in determining the causes of atopic dermatitis and respiratory allergies, especially in the light of the increasing prevalence and economic burden associated with these conditions. Globally, up to $20 \%$ of children suffer from atopic dermatitis [3]. According to the 2014 Global Asthma Report, it is estimated that 334 million people have asthma with $14 \%$ of the world's children and $8.6 \%$ of young adults exhibiting asthma symptoms [4]. Allergic rhinitis has a maximum global prevalence of $40 \%$. In the UK alone, the yearly financial burden of these three conditions is over $£ 2.5$ billion per year $[3,5-7]$. It is hoped that research into underpinning the causal mechanisms of atopic dermatitis and respiratory allergy can help to ameliorate the global burden of these conditions.

To date, the majority of research in understanding the link between atopic dermatitis and respiratory allergy has focused on establishing causal mechanisms via the existence of the supposed "atopic march" of allergic symptoms. The term "atopic march" refers to the temporal progression of symptoms during childhood from atopic dermatitis to asthma to allergic rhinitis $[2,8,9 \bullet, 10-14]$. This hypothesis is based on the observation that clinical signs of atopic dermatitis often occur earlier in life and often asthma and allergic rhinitis occur later on in life. This observation has widely led to the conclusion that atopic dermatitis is a causal "entry point" for subsequent asthma and allergic rhinitis [13]. Consequently, it has been suggested that effective management of atopic dermatitis could deter the progression of the atopic march, therefore preventing or at least reducing the subsequent development of asthma and allergic rhinitis. This conceptual framework has 
been the basis for studies aiming to prevent the development of airway inflammation and asthma in children who were deemed to be at risk by virtue of having atopic dermatitis and a family history of atopic disease [15•]. However, there is no evidence that targeting children with atopic dermatitis with an intervention designed to modify the progression towards asthma is effective [16].

The hypothesis of the atopic march as an explanation of the causal link between atopic dermatitis and respiratory allergies may be erroneously based on evidence on a population level without taking into account the profile of symptoms at the individual level. Using an epidemiological approach based on the prevalence of allergic diseases on a population level, we observe that atopic dermatitis has an increased prevalence in early life across the population followed by an increase in the prevalence of asthma with a subsequent increase in the prevalence of allergic rhinitis (Fig. 1a). Such an approach hinders a deeper understanding of the progression of symptoms at an individual level and determining whether such a progression of symptoms is indeed observed within individuals with an increased risk of atopic dermatitis in early life. Studies conducted investigating genetic and other biological markers of atopic dermatitis and other allergic diseases have raised the question as to whether atopic dermatitis is a cause of the subsequent development of asthma and allergic rhinitis or whether the presence of atopic dermatitis in early life is a consequence of distinct syndromes with different pathophysiological mechanisms for the subsequent development of respiratory allergy [13].

This article focuses on reviewing our current understanding of the link between atopic dermatitis and the subsequent progression of respiratory allergies during childhood and into early adolescence. When considering respiratory allergies, we will focus on asthma and rhinitis which are commonly described as allergic conditions. Indeed, the purpose of understanding allergic disease progression is with the view of developing targeted, personalised treatment and management strategies. Understanding the role of atopic dermatitis in the development of respiratory allergy may ultimately allow us to determine more effective treatment strategies, thus reducing the patient and economic burden associated with these conditions.

\section{Atopic Dermatitis and Respiratory Allergy: How are they Linked? Current Understanding in the Literature}

Several studies have hypothesised that the presence of atopic dermatitis within the first 2 years of life predicts subsequent asthma followed by rhinitis in later childhood $[8,9 \cdot, 10-14$, $15 \cdot]$. In the mid-twentieth century, this triad of symptoms was first hypothesised to be causally linked via the mechanism of the allergic or atopic march [17]. Later, at the beginning of the twenty-first century, with the increase in epidemiological studies, publications dealing with asthma and allergic diseases gave evidence at a population level to suggest a causal link between atopic dermatitis and subsequent asthma and allergic rhinitis.

Most of the studies which associated early-life atopic dermatitis with an increased risk of subsequent asthma and allergic rhinitis used cross-sectional statistical analysis techniques which do not take into account the temporal development of symptoms at a within-individual level $[8,11,17-24]$. The atopic march hypothesis has been criticised as an oversimplification of what happens to the disease profile on an individual

\section{A}

B
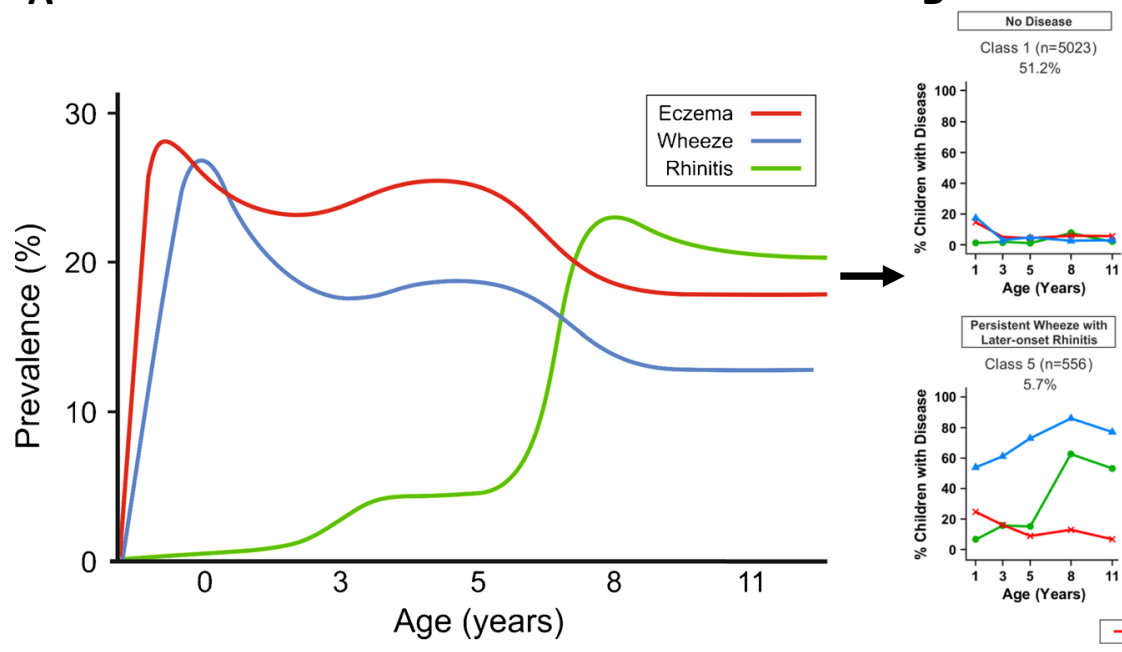

Fig. 1 Illustration showing that the atopic march may be the result of an ecological fallacy whereby the profiles of atopic dermatitis (eczema) wheeze and rhinitis follow a profile similar to the atopic march when looking at prevalence at a population level (a) [32•]. However, when we disaggregate profiles of symptoms at an individual level, we identify

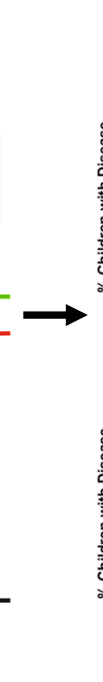

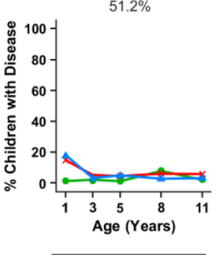

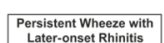
Later-onset Rhinitis $5.7 \%$

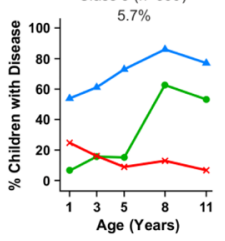
Study (MAAS)
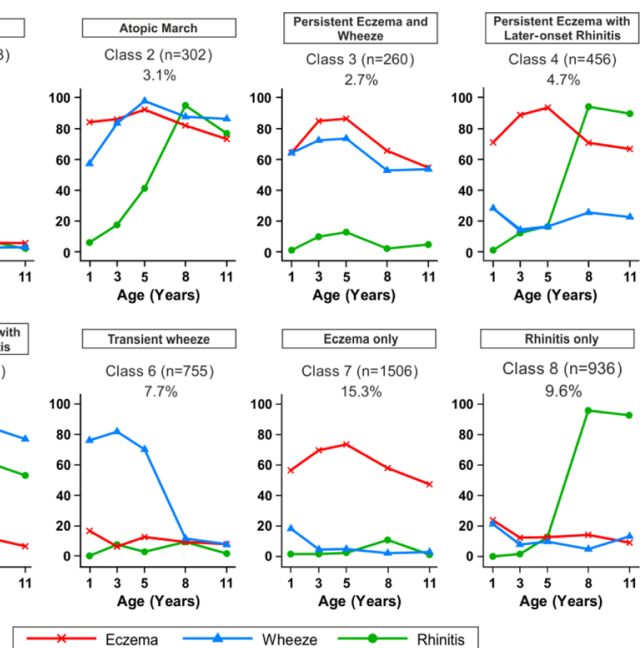

eight distinct profiles of co-occurrence of eczema, wheeze and rhinitis (b) [32•]. Data is derived from joint profiles of the Avon Longitudinal Study of Parents and Children (ALSPAC) and Manchester Asthma and Allergy 
level [25-28], and some studies have suggested that among patients there is heterogeneity in the chronology of the development of symptoms [28-30, 31・, 32•, 33]. Children with early atopic dermatitis who later develop asthma and rhinitis may belong to a distinct phenotype, rather than represent the typical progression of atopic diseases within the general population $[28,30]$. Despite apparent clear temporal association linking atopic dermatitis with progression to asthma and allergic rhinitis, there is no definitive proof that atopic march is causal or that atopic dermatitis is causal for the subsequent development of asthma and rhinitis [31•]. As there is little novelty in the conclusions and statistical-epidemiological methods used to assess whether atopic dermatitis is causally related to the subsequent development of asthma followed by allergic rhinitis via an atopic march of these symptoms, we merely acknowledge this as a widely hypothesised causal mechanism which has already been extensively reviewed in the literature [32•,33]. In the following part of this section, we will highlight studies which have utilised more novel approaches to understanding the underlying heterogeneity in the manifestation of atopic dermatitis and respiratory allergy in childhood.

A recent study from the ORCA Cohort applied cluster analysis to evaluate whether distinct phenotypes of earlyonset atopic dermatitis had different risk associated with the subsequent development of asthma [34]. Cluster analysis on 214 children with early-onset atopic dermatitis revealed two phenotypes of disease progression: a phenotype of multiple sensitisations (39\%) and another phenotype predominantly linked with family history of asthma (17\%), whereas $44 \%$ of these children did not go on to develop subsequent asthma/ allergy. Therefore, among children with early-onset atopic dermatitis, where we would expect the atopic march to be more pronounced, nearly half of these children do not develop the subsequent symptoms associated with the atopic march. The study concludes that the hypothesis that a "single entry" mechanism whereby atopic dermatitis leads to the subsequent development of asthma and allergic rhinitis is observed in a subgroup of children with multiple sensitisation. Therefore there may exist various pathophysiological mechanisms which lead to the atopic march progression from atopic dermatitis to asthma and then allergic rhinitis and different pathways of progression of allergic disease may be associated to different genetic, environmental and genetic-environmental influences.

A study of $\sim 310,000$ children born in Taiwan [35] evaluated the different courses of atopic dermatitis and their relationships to subsequent asthma and allergic rhinitis. This study superimposed phenotypes defined by Illi et al. [36] for subtypes of atopic dermatitis and phenotypes defined by Martinez et al. [37•] for different subtypes of wheeze. This approach led them to conclude that although the profile was similar to previous studies, the paradigm of the atopic march may have been an oversimplification of the profile that exists on an individual level with different atopic dermatitis phenotypes having significantly different risks of subsequent development of asthma and allergic rhinitis.

In a recent study [32•], we jointly modelled longitudinal data from two independent population-based birth cohorts: The Avon Longitudinal Study of Parents and Children (ALSPAC) Cohort and the Manchester Asthma and Allergy Study (MAAS) Cohort. The aim of this study was to investigate whether the atopic march profile which is observed on a population level can be observed on a within-individual level. This study consisted of atopic dermatitis, wheeze and rhinitis data from both cohorts which was collected from each child at ages 1, 3, 5, 8 and 11 years of age. This was the first study to investigate the presence of distinct individual-level profiles using a machine learning approach to infer the presence of different hypothetical latent classes which represent different profiles of atopic dermatitis, wheeze and rhinitis over time. In this study, we identified eight different classes describing distinct subgroups of temporal trajectories of atopic dermatitis, wheeze and rhinitis during childhood. Based on the characteristics of these classes, they were labelled as: no disease $(51.3 \%)$, atopic march $(3.1 \%)$, persistent atopic dermatitis and wheeze $(2.7 \%)$, persistent atopic dermatitis with lateronset rhinitis $(4.7 \%)$, persistent wheeze with later-onset rhinitis $(5.7 \%)$, transient wheeze (7.7\%), atopic dermatitis only $(15.3 \%)$ and rhinitis only $(9.6 \%)$. Less than $7 \%$ of children with symptoms had profiles similar to those of the atopic march, in which early atopic dermatitis and wheeze were followed by rhinitis. The remaining disease classes were generally characterised by the presence of only one or two of the three symptoms, indicating that more than $90 \%$ of children with symptoms commonly associated with atopy in childhood do not follow the widely perceived trajectory of the atopic march. Furthermore, highly concordant patterns of sensitisation over time were associated with very different profiles of symptoms. Conversely, for the three classes characterised by persistence of wheeze (atopic march, persistent atopic dermatitis and wheeze, persistent wheeze with later-onset rhinitis) patterns of sensitisation were different, as were patterns of comorbid atopic dermatitis and rhinitis. This may give evidence to suggest that atopic dermatitis, asthma and allergic rhinitis are all common and so co-exist, but mostly as independent entities. Although there is a group of children with trajectories similar to the atopic march paradigm and with a high degree of allergic sensitisation, this only comprises approximately one child in every 20 with symptoms.

In this study [32•], we also explicitly modelled the assumptions underlying the atopic march (that the profile of this march of symptoms may follow a chronological manifestation of atopic dermatitis, wheeze and then rhinitis) and found that, using the mathematical criteria of model evidence which is a measure of model likelihood, this model does not capture 
patterns in the data as well as a model in which we assumed that atopic dermatitis, wheeze and rhinitis are independent. This suggested that a profile of symptoms where we assume that atopic dermatitis, asthma and allergic rhinitis are distinct, independent diseases with different patterns of co-occurrence better encapsulated the profile of symptoms on a population level, than a model where we assume that the probability of developing subsequent respiratory allergy is conditioned on prior symptoms of atopic dermatitis. These distinct profiles with different associated factors may suggest distinct mechanisms: one which leads to atopic sensitization and those leading to symptoms. This is consistent with recent discovery of different phenotypes of atopy characterised by different patterns of IgE responses to allergens (both over time, and to different individual allergens), which differ in their associations with clinical symptoms [38・, 39, 40].

Previous studies which suggest evidence of an atopic march of symptoms from atopic dermatitis to respiratory allergy may be a have a flawed approach as they reflect patterns at the population level, rather than the natural covariance of symptoms within individuals' life courses. A recent review has challenged the lack of robust evidence for inferring that atopic dermatitis is causally linked to asthma and rhinitis [31•]. The link between atopic dermatitis and respiratory allergy may not be causal, but may rather be explained by an underlying latent (non-observed) disease status within the population, whose structure we were able to infer by investigating individual longitudinal disease profiles.

Although generally acknowledged that children with atopic dermatitis are at an increased risk of respiratory allergic diseases and atopic sensitisation, there is a need to move away from the traditional atopic march paradigm. The observed atopic march may be the result of an ecological fallacy whereby we observe a global prevalence profile of atopic dermatitis followed by asthma followed by allergic rhinitis at a population level, however, this profile of symptoms may not be observed when we look at trajectories of disease within individuals. Figure 1 illustrates where such a phenomenon occurs with the atopic march where at a population level, we observe profiles similar to those of the atopic march (Fig. 1a). However, using latent variable modelling, we identify distinct phenotypes with distinct profiles of co-occurrence between eczema, wheeze and rhinitis and only a subgroup of these profiles follow those similar to the atopic march. Most children with early atopic dermatitis will not develop asthma. Rather, there is a need for clinicians to acknowledge the heterogeneity of patterns of "allergic" diseases and to communicate to parents the uncertainty inherent in predicting the development of new symptoms or resolution of existing ones. This is a more realistic approach involving the disaggregation of a complex phenotype with the aim to understand different mechanisms associated with the subsequent development of asthma and allergic diseases. This would lead us towards a better understanding of different causes of heterogeneous pathways for asthma and allergic disease development.

\section{Statistical Machine Learning to Refine our Understanding of Distinct Disease Phenotypes}

Recent developments in machine learning provide new ways to capture heterogeneity in longitudinal patterns of distinct symptom categories within individual patients, where conventional modelling techniques might over-aggregate the underlying disease complexity [41]. Machine learning allows us to utilise a data-driven approach to identify structure within the data. Latent variable modelling is one such method of pattern recognition which can be applied to classify homogenous group patients. These methods are used commonly by computer scientists for problem-solving in many other fields and are increasingly used to disaggregate complex disease phenotypes in respiratory medicine and allergy [32•, 38•, 39-43]. Methodological development within this area can greatly enhance our ability to understand asthma and allergic disease heterogeneity in order to discover latent phenotypes which are replicable across different populations. It is hoped that such phenotypes will be clinically meaningful and have practical implications for our understanding of these conditions and how they are managed in the future.

The use of latent variable modelling strategies has been crucial in helping to disaggregate complex phenotypes where oversimplification of statistical associations may be an impediment to understanding underlying causal factors. An example of such a disaggregation of a complex phenotype is in the case of asthma, where latent variable modelling was used to understand the different subtypes of wheezing illness which have been placed under the umbrella term of asthma [44-46]. This led to understanding that asthma, rather than being a single disease, is an umbrella term used to define multiple conditions which display similar symptoms but have different triggers and manifestations over time. In order to identify causal associations and understand the co-existence of atopic dermatitis and respiratory allergy, a more refined phenotypic definition is a fundamental ingredient. Such a phenotypic definition will enhance our current understanding of the within-person variability in disease atopic dermatitis, asthma and rhinitis profiles and between-patient heterogeneity in disease development. These modelling strategies provide a flexible modelling framework whereby we can incorporate appropriate levels of model complexity and at the same time use a data-driven rather than hypothesis testing approach for developing model inferences to better understand disease development over time.

Together with enhanced statistical machine learning modelling frameworks which allow us to incorporate appropriate levels of complexity and uncertainty in discovering heterogeneous phenotypes, medical data science is rapidly moving towards a systems approach to understand the underlying causality of disease heterogeneity. A systems approach may 
lead to a better understanding of disease heterogeneity through multi-scale subtype discovery based on clinical data to incorporate genetic, biological and molecular data using an integrative systems biology approach to endotype discovery. Such an approach provides many methodological challenges due to the data complexity and computational expense involved in such models. However, it is worth pursuing this area of research, not only within allergy and respiratory medicine, but such approaches will be applicable to a more generalised medical framework for understanding disease complexity in other areas as well.

\section{Biomarkers as a Tool for Identifying Distinct Phenotypes}

There is a need for diagnostic tools such as biomarkers which can be used to predict different causal mechanisms and manifestation of symptoms. Given our understanding that the cooccurrence of atopic dermatitis, asthma and allergic rhinitis may have heterogeneous manifestations, some of which may resemble the profile of the hypothesised atopic march, future work may be carried out to investigate whether there are underlying biological and genetic markers indicating distinct mechanisms for these heterogeneous profiles of symptoms.

A recent review [44] highlighted the fact that the increasing prevalence of atopic dermatitis in the global population was suggestive that atopic dermatitis is not only due to heritability, but that there are other environmental effects as well as genetic factors that are modified by the environment (gene-environment interactions). As with many other studies [28, 30, 31•, 32•, 33], this review also highlighted the importance of understanding different subgroups of atopic dermatitis which have different subsequent associations with asthma and allergic rhinitis.

Unravelling the complex genetic architecture underlying atopic dermatitis and respiratory allergy and the different profiles of co-manifestation of these symptoms is an important premise for future therapies and potentially disease-modifying strategies [47]. However, because of the high degree of stratification suggested by the large number of heterogeneous profiles, identification of distinct biomarkers and implementation of more targeted treatment and management interventions may prove challenging due to a reduced sample size, but may also prove more effective due to a more accurate definition of distinct disease phenotypes.

One potential biomarker for the development of the atopic march profile of symptoms has been thymic stromal lymphopoietin (TSLP) [48]. Early presence of circulating TSLP was significantly associated with reduced incidence of recurrent wheeze in those children not sensitised to aeroallergen. These findings suggest a possible underlying distinction between pathogenesis of developing atopic vs. non-atopic recurrent wheeze. This may also be indicative of an uncoupling of mechanisms atopic dermatitis associated with different pathways, some of which are not causal. Previous studies: These findings raise the hypothesis that a cytokine (presumably TSLP) produced by the skin acts as a messenger to prime lung responses to allergen. If so, TSLP would be an excellent therapeutic target to reduce the incidence of asthma in children with a history of AD [48-51].

Another potential biomarker has been investigating the role of fillaggrin in the subsequent development of atopic dermatitis and subsequent atopic asthma and allergic rhinitis and whether this plays a role in children who exhibit a symptom profile similar to that of the atopic march [33, 52].

\section{Conclusions}

The emphasis on the "atopic march" without robust evidence to support this notion may hamper research into the underpinning the pathophysiology of atopy-related diseases [32•]. The current evidence suggests that the developmental profiles of atopic dermatitis, asthma and rhinitis are heterogeneous and that a small proportion of children with co-morbid symptoms follow a trajectory profile similar to that of the atopic march.

Two important lessons can be drawn from the review of the literature and should be taken as a general backdrop for research when assessing the link between atopic dermatitis and respiratory allergy. Firstly, although we acknowledge that there is a strong association between atopic dermatitis and respiratory allergy, association is not a sufficient condition to imply that these conditions are causally linked. Secondly, given the distinct observed profiles of atopic dermatitis, asthma and allergic rhinitis, we can infer that the manifestations of allergic symptom development over time are heterogeneous. This is a fundamental step towards understanding the causes of respiratory allergy. The distinct subgroups of symptom profiles may represent distinct causal mechanisms of allergic disease. The next phase of research on understanding the link between atopic dermatitis and respiratory allergies should be focused on a more unified approach to replicate endotypes discovered and to identify biomarkers for these distinct symptom profiles.

\section{Compliance with Ethics Guidelines}

Conflict of Interest Angela Simpson and Iain E. Buchan declare that they have no conflict of interest.

Danielle C. M. Belgrave reports personal fees from GSK and currently is an employee of GSK. The opinions expressed in this article are personal and in no way reflects the opinion of GSK and is in no way related to this employment.

Adnan Custovic reports personal fees from Novartis, Thermo Fisher, AstraZeneca, ALK, fees GlaxoSmithKline, and Regeneron/Sanofi.

Human and Animal Rights and Informed Consent This article does not contain any studies with human or animal subjects performed by any of the authors. 


\section{References}

Papers of particular interest, published recently, have been highlighted as:

- Of importance

1. Galli E, Gianni S, Auricchio G, Brunetti E, Mancino G, Rossi P. Atopic dermatitis and asthma. Allergy Asthma Proc. 2007;28(5): 540-3.

2. Spergel JM. From atopic dermatitis to asthma: the atopic march. Ann Allergy Asthma Immunol. 2010;105(2):99-106.

3. DaVeiga SP. Epidemiology of atopic dermatitis: a review. Allergy Asthma Proc. 2012;33(3):227-34.

4. The Global Asthma Report 2014. Auckland, New Zealand: Global Asthma Network, 2014.

5. Burns T, Breathnach S, Cox N, Griffiths C. Textbook of dermatology. Blackwell. 2004;49:32-49.

6. http://www.worldallergy.org/professional/allergic_diseases_center/ rhinitis/rhinitissynopsis.php]

7. Boyd R, Cowie H, Hurley F, Ayres J. The true cost of occupational asthma in Great Britain. Health Saf Exec. 2006;474:1-122.

8. Wahn U. What drives the allergic march? Allergy. 2000;55:591-9.

9. Wahn U, Bergmann R, Kulig M, Forster J, Bauer CP. The natural course of sensitisation and atopic disease in infancy and childhood. Pediatr Allergy Immunol. 1997;8:16-20. One of the first epidemiological studies to investigate the existence of the atopic march using longitudinal data.

10. Saunes M, Oien T, Dotterud CK, Romundstad PR, Storro O, et al. Early eczema and the risk of childhood asthma: a prospective, population-based study. BMC Pediatr. 2012;12:168.

11. Burgess JA, Lowe AJ, Matheson MC, Varigos G, Abramson MJ, et al. Does eczema lead to asthma? J Asthma. 2009;46:429-36.

12. Spergel JM. Epidemiology of atopic dermatitis and atopic march in children. Immunol Allergy Clin N Am. 2010;30:269-80.

13. Spergel JM, Paller AS. Atopic dermatitis and the atopic march. J Allergy Clin Immunol. 2003;112:S118-27.

14. Rhodes HL, Thomas P, Sporik R, Holgate ST, Cogswell JJ. A birth cohort study of subjects at risk of atopy: twenty-two-year follow-up of wheeze and atopic status. Am J Respir Crit Care Med. 2002;165: 176-80.

15. Pinart M, Benet M, Annesi-Maesano I, von Berg A, Berdel D, et al. Comorbidity of eczema, rhinitis, and asthma in IgE-sensitised and non-IgE-sensitised children in MeDALL: a population-based cohort study. Lancet Respir Med. 2014;2(2):131-40. Epidemiological study which differentiates possible IgEmediated and non-IgE-mediated co morbidity of asthma, wheeze and rhinitis.

16. Wahn P. Allergic factors associated with the development of asthma and the influence of cetirizine in a double-blind, randomised, placebo-controlled trial: First results of ETAC ${ }^{\circledR}$. Pediatr Allergy Immunol. 1998;9(3):116-24.

17. van der Hulst AE, Klip H, Brand PL. Risk of developing asthma in young children with atopic eczema: a systematic review. J Allergy Clin Immunol. 2007;120(3):565-9.

18. Saarinen UM, Kajosaari M. Breastfeeding as prophylaxis against atopic disease: prospective follow-up study until 17 years old. Lancet. 1995;346:1065-9.

19. Austin JB, Kaur B, Anderson HR, Burr M, Harkins LS, et al. Hay fever, eczema, and wheeze: a nationwide UK study (ISAAC, international study of asthma and allergies in childhood). Arch Dis Child. 1999;81:225-30.
20. Kurukulaaratchy RJ, Matthews S, Arshad SH. Defining childhood atopic phenotypes to investigate the association of atopic sensitization with allergic disease. Allergy. 2005;60:1280-6.

21. Martin PE, Matheson MC, Gurrin L, Burgess JA, Osborne N, et al. Childhood eczema and rhinitis predict atopic but not nonatopic adult asthma: a prospective cohort study over 4 decades. J Allergy Clin Immunol. 2011;127:1473-9.

22. von Kobyletzki LB, Bornehag CG, Hasselgren M, Larsson M, Lindstrom CB, et al. Eczema in early childhood is strongly associated with the development of asthma and rhinitis in a prospective cohort. BMC Dermatol. 2012;12:1471-5945.

23. Wuthrich B, Schmid-Grendelmeier P. The atopic eczema/dermatitis syndrome. Epidemiology, natural course, and immunology of the IgE-associated ("extrinsic") and the nonallergic ("intrinsic") AEDS. J Investig Allergol Clin Immunol. 2003;13:1-5.

24. Kulig M, Bergmann R, Niggemann B, Burow G, Wahn U. Prediction of sensitization to inhalant allergens in childhood: evaluating family history, atopic dermatitis and sensitization to food allergens. The MAS Study Group. Multicentre Allergy Study. Clin Exp Allergy. 1998;28:1397-403.

25. Hopper JL, Bui QM, Erbas B, Matheson MC, Gurrin LC, et al. Does eczema in infancy cause hay fever, asthma, or both in childhood? Insights from a novel regression model of sibling data. J Allergy Clin Immunol. 2012;130:1117-22.

26. Illi S, von Mutius E, Lau S, Nickel R, Niggemann B, et al. The pattern of atopic sensitization is associated with the development of asthma in childhood. J Allergy Clin Immunol. 2001;108:709-14.

27. van der Hulst AE, Klip H, Brand PL. Risk of developing asthma in young children with atopic eczema: a systematic review. J Allergy Clin Immunol. 2007;120:565-9.

28. Williams H, Flohr C. How epidemiology has challenged 3 prevailing concepts about atopic dermatitis. J Allergy Clin Immunol. 2006;118:209-13.

29. Barberio G, Pajno GB, Vita D, Caminiti L, Canonica GW, et al. Does a 'reverse' atopic march exist? Allergy. 2008;63:1630-2.

30. Illi S, von Mutius E, Lau S, Nickel R, Gruber C, et al. The natural course of atopic dermatitis from birth to age 7 years and the association with asthma. J Allergy Clin Immunol. 2004;113:925-31.

31. Dharmage SC, Lowe AJ, Matheson MC, Burgess JA, Allen KJ, et al. Atopic dermatitis and the atopic march revisited. Allergy. 2014;69:17-27. Comprehensive review article which offers a critical evaluation of the atopic march.

32. Belgrave DC, Granell R, Simpson A, Guiver J, Bishop C, et al. Developmental profiles of eczema, wheeze, and rhinitis: two population-based birth cohort studies. PLoS Med. 2014;11(10): e1001748. First paper to present a machine-learning approach to disaggregate distinct individual-level profiles of eczema, wheeze and rhinitis. This paper may have important consequences for understanding different mechanisms associated with different profiles of symptoms.

33. Zheng T, Yu J, Oh MH, Zhu Z. The atopic march: progression from atopic dermatitis to allergic rhinitis and asthma. Allergy Asthma Immunol Res. 2011;3(2):67-73.

34. Amat F, Saint-Pierre P, Bourrat E, Nemni A, Couderc R, et al. Early-onset atopic dermatitis in children: which are the phenotypes at risk of asthma? Results from the ORCA cohort. PLoS One. 2015;10(6):e0131369.

35. Shen CY, Lin MC, Lin HK, Lin CH, Fu LS, Fu YC. The natural course of eczema from birth to age 7 years and the association with asthma and allergic rhinitis: a population-based birth cohort study. Allergy Asthma Proc. 2013;34(1):78-83.

36. Illi S, von Mutius E, Lau S, Nickel R, Grüber C, et al. The natural course of atopic dermatitis from birth to age 7 years and the association with asthma. J Allergy Clin Immunol. 2004;113(5):925-31.

37. Martinez FD, Wright AL, Taussig LM, Holberg CJ, Halonen M, Morgan WJ. Asthma and wheezing in the first six years of life. N 
Engl J Med. 1995;332(3):133-8. Seminal paper which makes the first proposal of the need to dissagregate complex phenotypes of asthma and allergic disease.

38. Simpson A, Tan VY, Winn J, Svensen M, Bishop CM, et al. Beyond atopy: multiple patterns of sensitization in relation to asthma in a birth cohort study. Am J Respir Crit Care Med. 2010;181:1200-6. First paper to use a machine-learning approach to disaggregate complexities of allergic disease.

39. Lazic N, Roberts G, Custovic A, Belgrave D, Bishop CM, et al. Multiple atopy phenotypes and their associations with asthma: similar findings from two birth cohorts. Allergy. 2013;68:764-70.

40. Custovic A, Lazic N, Simpson A. Pediatric asthma and development of atopy. Curr Opin Allergy Clin Immunol. 2013;13:173-80.

41. Bishop CM. Model-based machine learning. Phil Trans R Soc A: Math Phys Eng Sci 371. 2013.

42. Prosperi MC, Belgrave D, Buchan I, Simpson A, Custovic A. Challenges in interpreting allergen microarrays in relation to clinical symptoms: a machine learning approach. Pediatr Allergy Immunol. 2014;25:71-9.

43. Prosperi MC, Sahiner UM, Belgrave D, Sackesen C, Buchan IE, et al. Challenges in identifying asthma subgroups using unsupervised statistical learning techniques. Am J Respir Crit Care Med. 2013; 188:1303-12.

44. Henderson J, Granell R, Heron J, Sherriff A, Simpson A. et al. Associations of wheezing phenotypes in the first six years of life with atopy, lung function and airway responsiveness in mid childhood. Thorax. 2008;63:974-80. doi:10.1136/thx.2007.093187.

45. Belgrave DC, Simpson A, Semic-Jusufagic A, Murray CS, Buchan I, Pickles A, et al. Joint modeling of parentally reported and physician-confirmed wheeze identifies children with persistent troublesome wheezing. J Allergy Clin Immunol. 2013;132(3): 575-83.

46. Belgrave DC, Custovic A, Simpson A. Characterizing wheeze phenotypes to identify endotypes of childhood asthma, and the implications for future management. Expert Rev Clin Immunol. 2013;9(10):921-36. doi:10.1586/1744666X.2013.836450.

47. Hoffjan S, Stemmler S. Unravelling the complex genetic background of atopic dermatitis: from genetic association results towards novel therapeutic strategies. Arch Dermatol Res. 2015;307(8):659-70. doi:10.1007/s00403-015-1550-6.

48. Bieber T, Cork M, Reitamo S. Atopic dermatitis: a candidate for disease-modifying strategy. Allergy. 2012;67(8):969-97.

49. Zhang Z, Hener P, Frossard N, Kato S, Metzger D, Li M, et al. Thymic stromal lymphopoietin overproduced by keratinocytes in mouse skin aggravates experimental asthma. Proc Natl Acad Sci U S A. 2009;106:1536-41.

50. Li M, Hener P, Zhang Z, Ganti KP, Metzger D, Chambon P. Induction of thymic stromal lymphopoietin expression in keratinocytes is necessary for generating an atopic dermatitis upon application of the active vitamin D3 analogue MC903 on mouse skin. J Investig Dermatol. 2009;129:498-502.

51. Demehri S, Morimoto M, Holtzman MJ, Kopan R. Skin-derived TSLP triggers progression from epidermal-barrier defects to asthma. PLoS Biol. 2009;7:e1000067.

52. Shaker M. New insights into the allergic march. Curr Opin Pediatr. 2014;26(4):516-20. 